
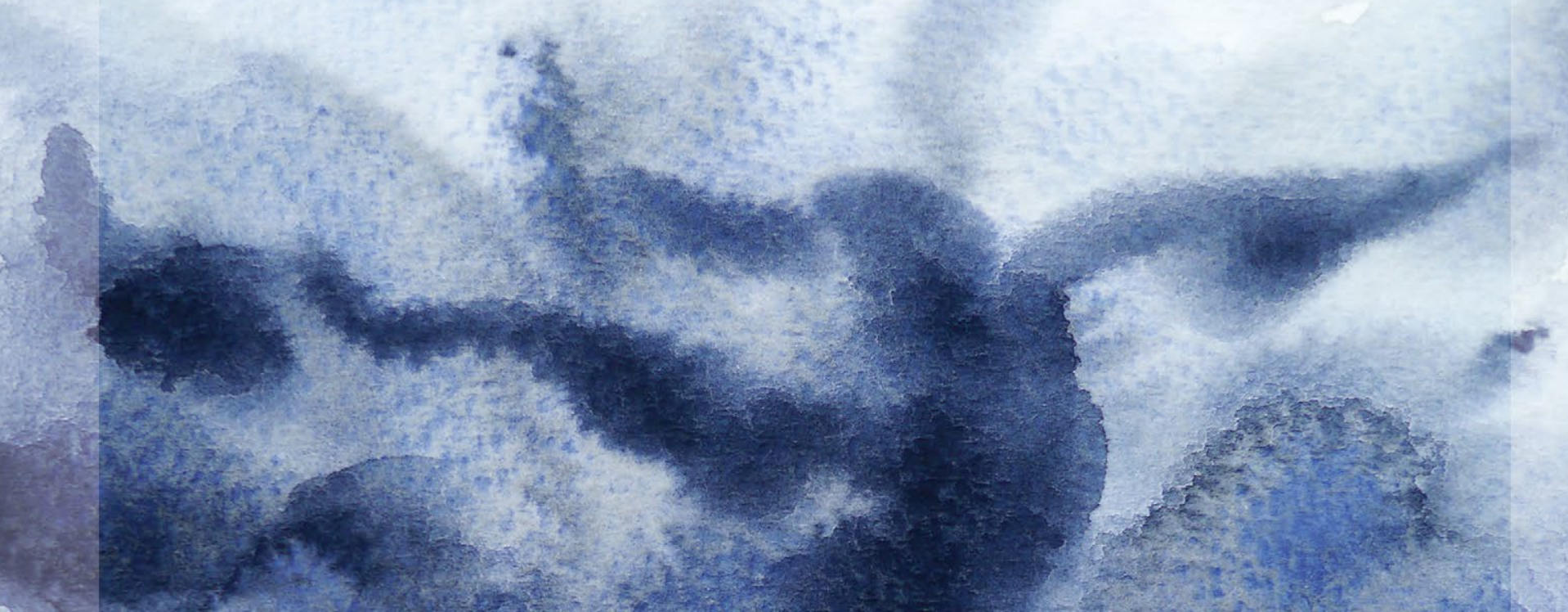
$x^{2} \times 2$

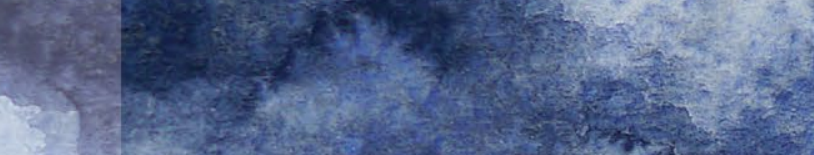

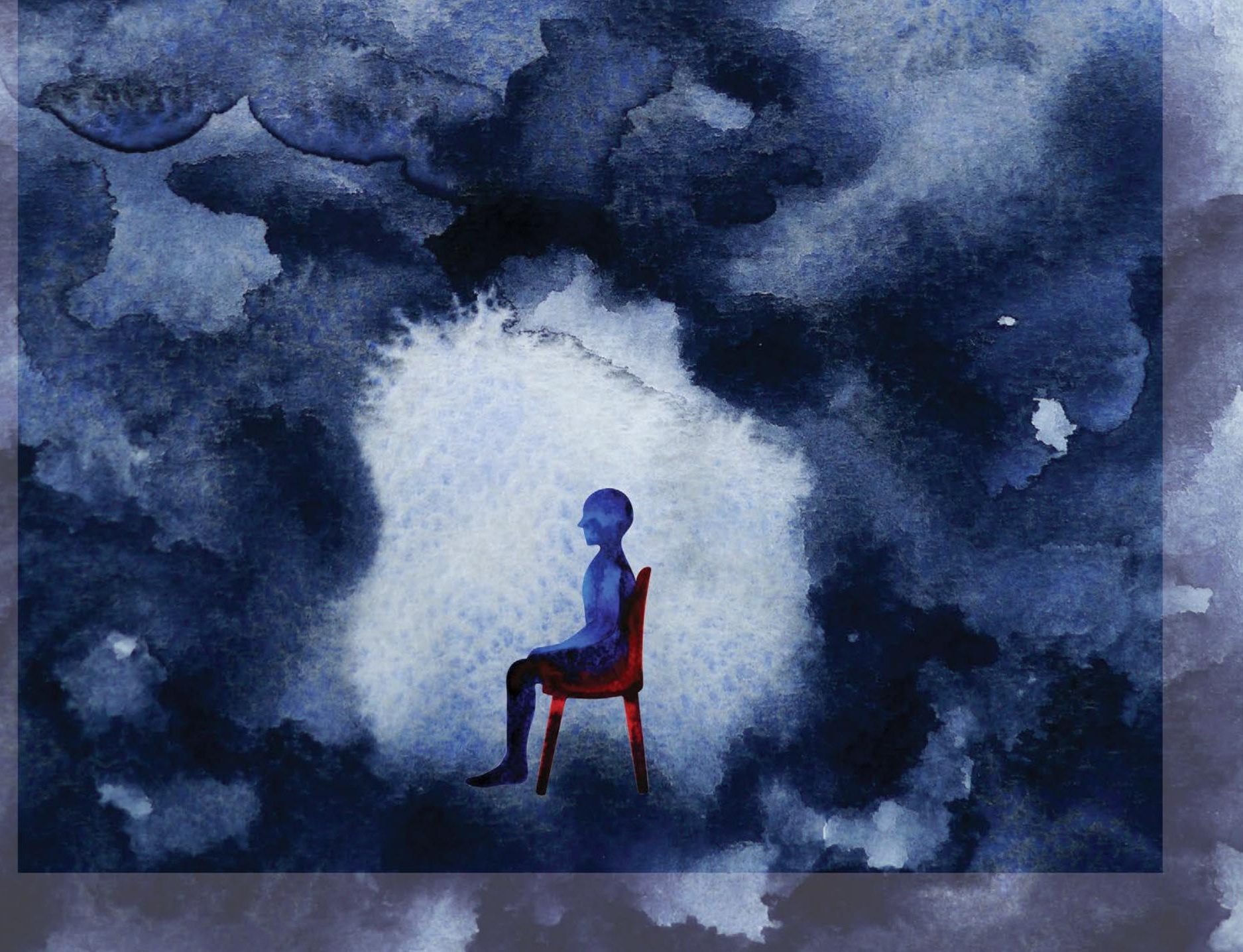




\section{FIRST OF 2 PARTS}

\section{Nontraditional therapies for treatment-resistant depression}

\section{Some off-label agents show promise, but carry risks}

\section{Mehmet E. Dokucu, MD, PhD \\ Associate Professor \\ Department of Psychiatry and Behavioral Sciences Northwestern University Feinberg School of Medicine Chicago, Illinois}

\section{Philip G. Janicak, MD}

Adjunct Professor

Department of Psychiatry and Behavioral Sciences Northwestern University Feinberg School of Medicine Chicago, Illinois

\section{Disclosures}

Dr. Dokucu reports no financial relationships with any companies whose products are mentioned in this article, or with manufacturers of competing products. Dr. Janicak is a speaker for Otsuka PsychU program and TMS Health Solutions.

doi: 10.12788/cp.0166 resently, FDA-approved first-line treatments and standard adjunctive strategies (eg, lithium, thyroid supplementation, stimulants, second-generation antipsychotics) for major depressive disorder (MDD) often produce less-than-desired outcomes while carrying a potentially substantial safety and tolerability burden. The lack of clinically useful and individual-based biomarkers (eg, genetic, neurophysiological, imaging) is a major obstacle to enhancing treatment efficacy and/or decreasing associated adverse effects (AEs). While the discovery of such tools is being aggressively pursued and ultimately will facilitate a more precision-based choice of therapy, empirical strategies remain our primary approach.

In controlled trials, several nontraditional treatments used primarily as adjuncts to standard antidepressants have shown promise. These include "repurposed" (off-label) medications, herbal/nutraceuticals, anti-inflammatory/immune system therapies, device-based treatments, and other alternative approaches.

Importantly, some nontraditional treatments also demonstrate AEs (Table, ${ }^{1-16}$ page 40). With a careful consideration of the risk/benefit balance, this article reviews some of the better-studied treatment options for patients with treatment-resistant depression (TRD). In Part 1, we will examine off-label medications. In Part 2, we will review other nontraditional approaches to TRD, including herbal/nutraceuticals, anti-inflammatory/immune system therapies, device-based treatments, and other alternative approaches.

We believe this review will help clinicians who need to formulate a different approach after their patient with depression is not helped 


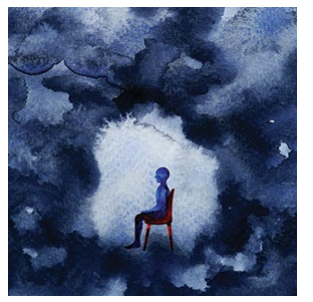

Nontraditional therapies for TRD

\section{Clinical Point}

In 3 RCTs, the evidence of efficacy of pimavanserin for patients whose depression did not respond to SSRls or SNRIs was mixed

\section{Risk levels and adverse effects of off-label medications for treatment-resistant depression}

\begin{tabular}{|c|c|}
\hline Treatment & Potential adverse effects \\
\hline \multicolumn{2}{|l|}{ Low risk } \\
\hline Pimavanserin ${ }^{1-3}$ & Dry mouth, nausea, headache \\
\hline Botulinum toxin ${ }^{10}$ & Local irritation at the injection site, transient headache \\
\hline \multirow[t]{2}{*}{$\mathrm{N}$-acetylcysteine ${ }^{16}$} & $\begin{array}{l}\text { Nausea, vomiting, diarrhea, constipation, musculoskeletal complaints, } \\
\text { decreased energy, headache }\end{array}$ \\
\hline & Rare: Rashes, fever, headache, drowsiness, low blood pressure, liver problems \\
\hline \multicolumn{2}{|l|}{ Medium risk } \\
\hline Antihypertensives ${ }^{4}$ & Headache, nausea, memory problems, cough, dizziness, hypotension \\
\hline Ketamine $e^{5-7}$ & $\begin{array}{l}\text { Drowsiness, dizziness, poor coordination, blurred vision, feeling strange or } \\
\text { unreal, hemodynamic change, psychotomimetic and dissociative symptoms } \\
\text { Risk for abuse }\end{array}$ \\
\hline \multicolumn{2}{|l|}{ High risk } \\
\hline Scopolamine $e^{8,9}$ & Agitation, dry mouth, urinary retention, cognitive clouding \\
\hline Mifepristone ${ }^{11}$ & $\begin{array}{l}\text { Gastrointestinal symptoms, headaches, dizziness } \\
\text { In clinical trials, } 3 \text { deaths occurred ( } 2 \text { patients who received mifepristone, } \\
1 \text { patient who received placebo) }\end{array}$ \\
\hline Estrogens ${ }^{12-14}$ & $\begin{array}{l}\text { Vaginal bleeding, dysmenorrhea, fibroid enlargement, galactorrhea, ovarian } \\
\text { cancer, endometrial cancer, breast cancer, deep vein thrombosis, pulmonary } \\
\text { embolism, hypertension, chest pain, myocardial infarction, stroke }\end{array}$ \\
\hline Buprenorphine ${ }^{15}$ & Nausea, vomiting, constipation, dizziness, somnolence, fatigue, sedation \\
\hline
\end{tabular}

by traditional first-, second-, and third-line treatments. The potential options discussed in Part 1 of this article are categorized based on their putative mechanism of action (MOA) for depression.

\section{Serotonergic and noradrenergic strategies}

Pimavanserin is FDA-approved for treatment of Parkinson's psychosis. Its potential MOA as an adjunctive strategy for MDD may involve 5-HT2A antagonist and inverse agonist receptor activity, as well as lesser effects at the 5-HT2C receptor.

A 2-stage, 5-week randomized controlled trial (RCT) (CLARITY; $\mathrm{N}=207$ ) found adjunctive pimavanserin $(34 \mathrm{mg} / \mathrm{d})$ produced a robust antidepressant effect vs placebo in patients whose depression did not respond to selective serotonin reuptake inhibitors (SSRIs) or serotonin-norepinephrine reuptake inhibitors (SNRIs). ${ }^{1}$ Furthermore, a secondary analysis of the data suggested that pimavanserin also improved sleepiness $(P<.0003)$ and daily functioning $(P<.014)$ at Week $5 .^{2}$

Unfortunately, two 6-week, Phase III RCTs (CLARITY-2 and $-3 ; \mathrm{N}=298$ ) did not find a statistically significant difference between active treatment and placebo. This was based on change in the primary outcome measure (Hamilton Depression Rating Scale-17 score) when adjunctive pimavanserin ( $34 \mathrm{mg} / \mathrm{d}$ ) was added to an SSRI or SNRI in patients with TRD. ${ }^{3}$ There was, however, a significant difference favoring active treatment over placebo based on the Clinical Global ImpressionSeverity score.

In these trials, pimavanserin was generally well-tolerated. The most common AEs were dry mouth, nausea, and headache. Pimavanserin has minimal activity at norepinephrine, dopamine, histamine, or acetylcholine receptors, thus avoiding AEs associated with these receptor interactions.

Given the mixed efficacy results of existing trials, further studies are needed to 
clarify this agent's overall risk/benefit in the context of TRD.

\section{Antihypertensive medications}

Emerging data suggest that some betaadrenergic blockers, angiotensin-inhibiting agents, and calcium antagonists are associated with a decreased incidence of depression. A large 2020 study $(\mathrm{N}=3,747,190)$ used population-based Danish registries (2005 to 2015) to evaluate if any of the 41 most commonly prescribed antihypertensive medications were associated with the diagnosis of depressive disorder or use of antidepressants. ${ }^{4}$ These researchers found that enalapril, ramipril, amlodipine, propranolol, atenolol, bisoprolol, carvedilol $(P<.001)$, and verapamil $(P<.004)$ were strongly associated with a decreased risk of depression. ${ }^{4}$

Adverse effects across these different classes of antihypertensives are well characterized, can be substantial, and commonly are related to their impact on cardiovascular function (eg, hypotension). Clinically, these agents may be potential adjuncts for patients with TRD who need antihypertensive therapy. Their use and the choice of specific agent should only be determined in consultation with the patient's primary care physician (PCP) or appropriate specialist.

\section{Glutamatergic strategies}

Ketamine is a dissociative anesthetic and analgesic. Its MOA for treating depression appears to occur primarily through antagonist activity at the $N$-methyl-D-aspartate ionotropic receptor of the glutamatergic system. There is preliminary evidence that its opioid receptor actions also may contribute to its antidepressant effect. ${ }^{5}$

Many published studies and reviews have described ketamine's role for treating MDD. Several studies have reported that low-dose $(0.5 \mathrm{mg} / \mathrm{kg})$ IV ketamine infusions can rapidly attenuate severe episodes of MDD as well as associated suicidality. For example, a meta-analysis of 9 RCTs (N = 368) comparing ketamine to placebo for acute treatment of unipolar and bipolar depression reported superior therapeutic effects with active treatment at 24 hours,
72 hours, and 7 days. ${ }^{6}$ The response and remission rates for ketamine were $52 \%$ and $21 \%$ at 24 hours; $48 \%$ and $24 \%$ at 72 hours; and $40 \%$ and $26 \%$ at 7 days, respectively. ${ }^{6}$

The most commonly reported AEs during the 4 hours after ketamine infusion included?:

- drowsiness, dizziness, poor coordination

- blurred vision, feeling strange or unreal

- hemodynamic changes (approximately $33 \%$ )

- small but significant $(P<.05)$ increases in psychotomimetic and dissociative symptoms.

Because some individuals use ketamine recreationally, this agent also carries the risk of abuse.

Research is ongoing on strategies for long-term maintenance ketamine treatment, and the results of both short- and long-term trials will require careful scrutiny to better assess this agent's safety and tolerability. Clinicians should first consider esketamine- the S-enantiomer of ketamine-because an intranasal formulation of this agent is FDA-approved for treating patients with TRD or MDD with suicidality when administered in a Risk Evaluation and Mitigation Strategy-certified setting.

\section{Cholinergic strategies}

Scopolamine is a potent muscarinic receptor antagonist used to prevent nausea and vomiting caused by motion sickness or medications used during surgery. Its use for MDD is based on the theory that muscarinic receptors may be hypersensitive in mood disorders.

Several double-blind RCTs of patients with unipolar or bipolar depression that used 3 pulsed IV infusions $(4.0 \mathrm{mcg} / \mathrm{kg}$ ) over 15 minutes found a rapid, robust antidepressant effect with scopolamine vs placebo. ${ }^{8,9}$ The oral formulation might also be effective, but would not have a rapid onset.

Common adverse effects of scopolamine include agitation, dry mouth, urinary retention, and cognitive clouding. Given scopolamine's substantial AE profile, it should be considered only for patients with TRD who could also benefit

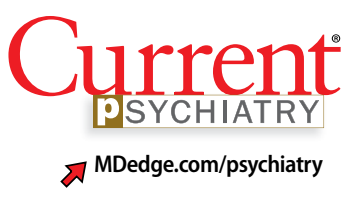

Clinical Point

Preliminary evidence suggests that ketamine's opioid receptor actions may contribute to its antidepressant effect 


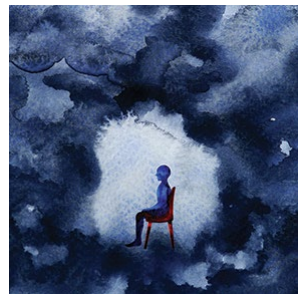

Nontraditional therapies for TRD

\section{Clinical Point}

\section{Botulinum toxin's mechanism of action for depression may involve its paralytic effects after injection into the glabella forehead muscle}

from the oral formulation for the medical indications noted above, should generally be avoided in older patients, and should be prescribed in consultation with the patient's PCP.

Botulinum toxin. This neurotoxin inhibits acetylcholine release. It is used to treat disorders characterized by abnormal muscular contraction, such as strabismus, blepharospasm, and chronic pain syndromes. Its MOA for depression may involve its paralytic effects after injection into the glabella forehead muscle (based on the facial feedback hypothesis), as well as modulation of neurotransmitters implicated in the pathophysiology of depression.

In several small trials, injectable botulinum toxin type A (BTA) (29 units) demonstrated antidepressant effects. A recent review that considered 6 trials $(\mathrm{N}=235$; 4 of the 6 studies were RCTs, 3 of which were rated as high quality) concluded that BTA may be a promising treatment for MDD. ${ }^{10}$ Limitations of this review included lack of a priori hypotheses, small sample sizes, gender bias, and difficulty in blinding.

In clinical trials, the most common AEs included local irritation at the injection site and transient headache. This agent's relatively mild AE profile and possible overlap when used for some of the medical indications noted above opens its potential use as an adjunct in patients with comorbid TRD.

\section{Endocrine strategies}

Mifepristone (RU486). This anti-glucocorticoid receptor antagonist is used as an abortifacient. Based on the theory that hyperactivity of the hypothalamic-pituitary-adrenal axis is implicated in the pathophysiology of MDD with psychotic features (psychotic depression), this agent has been studied as a treatment for this indication.

An analysis of 5 double-blind RCTs $(\mathrm{N}=1,460)$ found that 7 days of mifepristone, $1,200 \mathrm{mg} / \mathrm{d}$, was superior to placebo $(P<.004)$ in reducing psychotic symptoms of depression. ${ }^{11}$ Plasma concentrations $\geq 1,600 \mathrm{ng} / \mathrm{mL}$ may be required to maximize benefit. ${ }^{11}$

Overall, this agent demonstrated a good safety profile in clinical trials, with treatment-emergent AEs reported in 556 $(66.7 \%)$ patients who received mifepristone vs $386(61.6 \%)$ patients who received placebo. ${ }^{11}$ Common AEs included gastrointestinal (GI) symptoms, headache, and dizziness. However, 3 deaths occurred: 2 patients who received mifepristone and 1 patient who received placebo. Given this potential for a fatal outcome, clinicians should first consider prescribing an adjunctive antipsychotic agent or electroconvulsive therapy.

Estrogens. These hormones are important for sexual and reproductive development and are used to treat various sexual/reproductive disorders, primarily in women. Their role in treating depression is based on the observation that perimenopause is accompanied by an increased risk of new and recurrent depression coincident with declining ovarian function.

Evidence supports the antidepressant efficacy of transdermal estradiol plus progesterone for perimenopausal depression, but not for postmenopausal depression. ${ }^{12-14}$ However, estrogens carry significant risks that must be carefully considered in relationship to their potential benefits. These risks include:

- vaginal bleeding, dysmenorrhea

- fibroid enlargement

- galactorrhea

- ovarian cancer, endometrial cancer, breast cancer

- deep vein thrombosis, pulmonary embolism

- hypertension, chest pain, myocardial infarction, stroke.

The use of estrogens as an adjunctive therapy for women with treatment-resistant perimenopausal depression should only be undertaken when standard strategies have failed, and in consultation with an endocrine specialist who can monitor for potentially serious AEs.

\section{Opioid medications}

Buprenorphine is used to treat opioid use disorder (OUD) as well as acute and chronic pain. The opioid system is involved in the regulation of mood and may be an appropriate target for novel antidepressants. The use of buprenorphine 
in combination with samidorphan (a preferential mu-opioid receptor antagonist) has shown initial promise for TRD while

Although earlier results were mixed, a pooled analysis of 2 recent large RCTs $(\mathrm{N}=760)$ of patients with MDD who had not responded to antidepressants reported greater reduction in Montgomery-Åsberg Depression Rating Scale scores from baseline for active treatment (buprenorphine/ samidorphan; $2 \mathrm{mg} / 2 \mathrm{mg}$ ) vs placebo at multiple timepoints, including end of treatment $(-1.8 ; P<.010) .{ }^{15}$

The most common AEs included nausea, constipation, dizziness, vomiting, somnolence, fatigue, and sedation. There was minimal evidence of abuse, dependence, or opioid withdrawal. Due to the opioid crisis in the United States, the resulting relaxation of regulations regarding prescribing buprenorphine, and the high rates of depression among patients with OUD, buprenorphine/samidorphan, which is an investigational agent that is not FDAapproved, may be particularly helpful for patients with OUD who also experience comorbid TRD.

\section{Antioxidant agents}

$\mathrm{N}$-acetylcysteine (NAC) is an amino acid that can treat acetaminophen toxicity and moderate hepatic damage by increasing glutathione levels. Glutathione is also the primary antioxidant in the CNS. NAC may protect against oxidative stress, chelate heavy metals, reduce inflammation, protect against mitochondrial dysfunction, inhibit apoptosis, and enhance neurogenesis, all potential pathophysiological processes that may contribute to depression. ${ }^{16}$ minimizing abuse potential.

\section{Related Resource}

- Joshi KG, Frierson RL. Off-label prescribing: How to limit your liability. Current Psychiatry. 2020;19(9):12,39.

Drug Brand Names

\begin{tabular}{|c|c|}
\hline $\begin{array}{l}\text { Amlodipine } \cdot \text { Katerzia, } \\
\text { Norvasc }\end{array}$ & $\begin{array}{l}\text { Estradiol transdermal • } \\
\text { Estraderm }\end{array}$ \\
\hline Atenolol · Tenormin & Ketamine $\cdot$ Ketalar \\
\hline Bisoprolol · Zebeta & Mifepristone $\cdot$ Mifeprex \\
\hline $\begin{array}{l}\text { Buprenorphine - Sublocade, } \\
\text { Subutex }\end{array}$ & $\begin{array}{l}\text { Pimavanserin } \cdot \text { Nuplazid } \\
\text { Progesterone } \cdot \text { Prometrium }\end{array}$ \\
\hline Carvedilol · Coreg & Propranolol · Inderal \\
\hline Enalapril - Vasotec & Ramipril · Altace \\
\hline Esketamine - Spravato & Verapamil · Calan, Verelan \\
\hline
\end{tabular}

A systematic review and meta-analysis of 5 RCTs $(N=574)$ considered patients with various depression diagnoses who were randomized to adjunctive NAC, 1,000 mg twice a day, or placebo. Over 12 to 24 weeks, there was a significantly greater improvement in mood symptoms and functionality with NAC vs placebo. ${ }^{16}$

Overall, NAC was well-tolerated. The most common AEs were GI symptoms, musculoskeletal complaints, decreased energy, and headache. While NAC has been touted as a potential adjunct therapy for several psychiatric disorders, including TRD, the evidence for benefit remains limited. Given its favorable AE profile, however, and overthe-counter availability, it remains an option for select patients. It is important to ask patients if they are already taking NAC.

\section{Options beyond off-label medications}

There are a multitude of options available for addressing TRD. Many FDAapproved medications are repurposed and prescribed off-label for other indications when the risk/benefit balance is favorable.

continued on page 49

\section{Bottom Line}

Off-label medications that may offer benefit for patients with treatment-resistant depression (TRD) include pimavanserin, antihypertensive agents, ketamine, scopolamine, botulinum toxin, mifepristone, estrogens, buprenorphine, and $\mathrm{N}$-acetylcysteine. Although some evidence supports use of these agents as adjuncts for TRD, an individualized risk/benefit analysis is required.

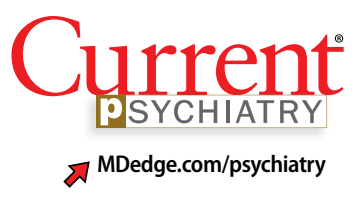

Clinical Point

In a meta-analysis of 5 RCTs, there was a significantly greater improvement in mood symptoms in patients taking NAC, $1,000 \mathrm{mg}$ twice a day 
Nontraditional therapies for TRD

continued from page 43

In Part 1 of this article, we reviewed several off-label medications that have supportive controlled data for treating TRD. In Part 2, we will review other nontraditional therapies for TRD, including herbal/nutraceuticals, antiinflammatory/immune system therapies, device-based treatments, and other alternative approaches.

\section{References}

1. Fava M, Dirks B, Freeman M, et al. A phase 2, randomized, double-blind, placebo-controlled study of adjunctive pimavanserin in patients with major depressive disorder and an inadequate response to therapy (CLARITY). J Clin Psychiatry. 2019;80(6):19m12928.

2. Tha MK, Fava M, Freeman MP, et al. Effect of adjunctive pimavanserin on sleep/wakefulness in patients with major depressive disorder: secondary analysis from CLARITY. J Clin Psychiatry. 2020;82(1):20m13425.

3. ACADIA Pharmaceuticals announces top-line results from the Phase 3 CLARITY study evaluating pimavanserin for the adjunctive treatment of major depressive disorder. News release. Acadia Pharmaceuticals Inc. Published July 20, 2020 https://ir.acadia-pharm.com/news-releases/news-releasedetails/acadia-pharmaceuticals-announces-top-line-resultsphase-3-0

4. Kessing LV,Rytgaard HC,EkstromCT,etal. Antihypertensive drugs and risk of depression: a nationwide populationbased study. Hypertension. 2020;76(4):1263-1279.

5. Williams NR, Heifets BD, Blasey C, et al. Attenuation of antidepressant effects of ketamine by opioid receptor antagonism. Am J Psychiatry. 2018;175(12):1205-1215.

6. Han Y, Chen J, Zou D, et al. Efficacy of ketamine in the rapid treatment of major depressive disorder: a meta-analysis of randomized, double-blind, placebo-controlled studies. Neuropsychiatr Dis Treat. 2016;12:2859-2867.
7. Wan LB, Levitch CF, Perez AM, et al. Ketamine safety and tolerability in clinical trials for treatment-resistant depression. J Clin Psychiatry. 2015;76(3):247-252.

8. Hasselmann, H. Scopolamine and depression: a role for muscarinic antagonism? CNS Neurol Disord Drug Targets. 2014;13(4):673-683.

9. Drevets WC, Zarate CA Jr, Furey ML. Antidepressant effects of the muscarinic cholinergic receptor antagonist scopolamine: a review. Biol Psychiatry. 2013;73(12): 1156-1163.

10. Stearns TP, Shad MU, Guzman GC. Glabellar botulinum toxin injections in major depressive disorder: a critical review. Prim Care Companion CNS Disord. 2018;20(5): $18 \mathrm{r} 02298$.

11. Block TS, Kushner H, Kalin N, et al. Combined analysis of mifepristone for psychotic depression: plasma levels associated with clinical response. Biol Psychiatry. 2018; 84(1):46-54.

12. Rubinow DR, Johnson SL, Schmidt PJ, et al. Efficacy of estradiol in perimenopausal depression: so much promise and so few answers. Depress Anxiety. 2015;32(8): 539-549.

13. Schmidt PJ, Ben Dor R, Martinez PE, et al. Effects of estradiol withdrawal on mood in women with past perimenopausal depression: a randomized clinical trial. JAMA Psychiatry. 2015;72(7):714-726.

14. Gordon JL, Rubinow DR, Eisenlohr-Moul TA, et al. Efficacy of transdermal estradiol and micronized progesterone in the prevention of depressive symptoms in the menopause transition: a randomized clinical trial. JAMA Psychiatry. 2018;75(2):149-157.

15. Fava M, Thase ME, Trivedi MH, et al. Opioid system modulation with buprenorphine/samidorphan combination for major depressive disorder: two randomized controlled studies. Mol Psychiatry. 2020;25(7): 1580-1591.

16. Fernandes BS, Dean OM, Dodd S, et al. N-Acetylcysteine in depressive symptoms and functionality: a systematic review and meta-analysis. J Clin Psychiatry. 2016;77(4): e457-466.

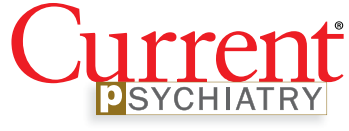

$\nabla^{\text {MDedge.com/psychiatry }}$

\section{Clinical Point}

Although some evidence supports these nontraditional therapies for TRD, an individualized risk-benefit analysis is required 